\title{
Extension of vector-valued holomorphic and harmonic functions
}

\author{
by \\ José Bonet (Valencia), Leonhard Frerick (Trier) and \\ ENRIQUe Jordá (Alcoy)
}

\begin{abstract}
We present a unified approach to the study of extensions of vector-valued holomorphic or harmonic functions based on the existence of weak or weak ${ }^{*}$-holomorphic or harmonic extensions. Several recent results due to Arendt, Nikolski, Bierstedt, Holtmanns and Grosse-Erdmann are extended. An open problem by Grosse-Erdmann is solved in the negative. Using the extension results we prove existence of Wolff type representations for the duals of certain function spaces.
\end{abstract}

1. Introduction. The purpose of this paper is to present a unified treatment of the extension of holomorphic or harmonic vector-valued functions, including the several variables case. Vector-valued holomorphic functions are useful in the theory of topological algebras [19] and of one-parameter semigroups [4, 18], in infinite-dimensional holomorphy [15, Chapter 3], and in operator theory $[1,20]$. Composition operators on spaces of this type have been investigated recently $[10,11,32,31]$. The topic we consider is closely related to the investigation of conditions ensuring that a weakly holomorphic function with values in a locally convex space is holomorphic. In fact, it is much easier to show that a function is weakly holomorphic and conclude that the original function is holomorphic as a consequence of an abstract theorem. The classical theorem of Dunford and Grothendieck shows that a function $f$ defined on an open set $\Omega \subseteq \mathbb{C}$ in the complex plane with values in a complete locally convex space $E$ is holomorphic if $u \circ f$ is holomorphic for every $u \in E^{\prime}$ in the topological dual of $E$. Several authors presented

2000 Mathematics Subject Classification: 46E40, 46A04.

Key words and phrases: vector-valued holomorphic functions, topological sheaves, Fréchet-Schwartz spaces, (DFS)-spaces, locally complete spaces.

The research of the first and third authors is supported by FEDER and MCYT, Proyecto no. MTM2004-02262. The research began during a stay of the third author at the Bergische Universität Wuppertal. The stay was supported by Generalitat Valenciana, Ayudas para estancias postdoctorales. Ref. CTESPP/2004/049. 
extensions of this result and related it to the extension of holomorphic functions; see Bogdanowicz [8], Colombeau [13] and Gramsch [21, 22]. Weak conditions for holomorphy of a vector-valued function have found renewed interest recently. Grosse-Erdmann [23] showed that it is enough to test weak holomorphy of a locally bounded function with values in a locally complete locally convex space on the elements of a separating subset of the dual of the range space, solving a problem posed by Wrobel [42]. Arendt and Nikolski [2] gave a short proof of this result if the range space is Fréchet; and GrosseErdmann [24] shortened his original proof with a more functional-analytic approach. He also treated holomorphic extension, and stated several open problems which we address in our article.

The basic problem we consider can be stated as follows: Let $\Omega$ be an open subset of $\mathbb{R}^{N}$ (or a smooth manifold), let $\mathscr{F}$ be a sheaf of smooth functions on $\Omega$, and let $f: M \rightarrow E$ be a function from a subset $M$ of $\Omega$ into a locally convex space $E$ such that $u \circ f$ has a unique extension $f_{u} \in \mathscr{F}(\Omega)$ for each $u$ in a separating subset of $E^{\prime}$. Does $f$ have an extension $\widehat{f}$ belonging to the space $\mathscr{F}(\Omega, E)$ of vector-valued $\mathscr{F}(\Omega)$-functions? We present theorems which simultaneously extend results due to Gramsch [22], Arendt and Nikolski [2] and Grosse-Erdmann [24]. Our approach using sheaves of smooth functions permits us to treat not only spaces defined on open subsets of the complex plane as in [2] and [24], but also holomorphic or harmonic functions of several variables and kernels of linear partial differential operators, thus including consequences of the work of Bierstedt and Holtmanns [7] and Enflo and Smithies [17]. Besides the positive results, we solve a problem of Grosse-Erdmann in the negative; see Example 20. Finally, we give representations of $\mathscr{F}^{\prime}(\Omega)$ and of $\mathscr{F}^{\prime}(\Omega, E)$ in the spirit of Wolff's description of the dual of the space of one variable holomorphic functions on a domain [41], used by Grosse-Erdmann to obtain the extension result [24, Theorem 2].

Our proofs are functional-analytic. They are based on properties of Fréchet-Schwartz spaces, the local completion of a locally convex space [36, Chapter 5], a theorem of Raikov about (DFS)-spaces (see e.g. [36, 8.5.28]), and the theory of $\varepsilon$-products of Schwartz [39]. In fact all the spaces of holomorphic or harmonic functions we are interested in are Fréchet-Schwartz spaces and their duals are (DFS)-spaces. These powerful abstract techniques have not been exploited before in this context. They permit us to derive many results with relatively smooth proofs.

\section{Preliminaries and notation}

2.1. $\varepsilon$-products and locally complete spaces. Our notation for locally convex spaces and functional analysis is standard. We refer the reader to $[27,29,33,36]$, and we recall some terminology. For a locally convex space $E$, 
which we assume to be Hausdorff, $E^{\star}$ and $E^{\prime}$ stand for its algebraic dual and topological dual, respectively. We denote by $\beta(E, F)$ the strong topology and by $\sigma(E, F)$ the weak topologies on $E$ with respect to a dual pair $\langle E, F\rangle$. As usual, if $E^{\prime}$ is the topological dual of a locally convex space $E$, the topology $\sigma\left(E^{\prime}, E\right)$ is called the weak ${ }^{\star}$ topology. We denote by co $=\operatorname{co}\left(E^{\prime}, E\right)$ the topology of uniform convergence on the compact and absolutely convex subsets of the locally convex space $E$. The polar in $E$ of a subset $A$ of $F$ in the dual pair $\langle E, F\rangle$ is $A^{\circ}:=\{x \in E:|f(x)| \leq 1$ for all $f \in A\}$. A subspace $G$ of $E^{\prime}$ is called separating if $u(x)=0$ for each $u \in G$ implies $x=0$. Clearly this is equivalent to $G$ being weak $^{\star}$-dense (or dense in the co-topology). If $E, F$ are locally convex spaces, then $L(E, F)$ denotes the vector space of all continuous linear maps from $E$ to $F$. Given $T \in L(E, F)$ we denote by $T^{t} \in L\left(F^{\prime}, E^{\prime}\right)$ its transpose defined by $T^{t}(u)=u \circ T \in E^{\prime}$ for each $u \in F^{\prime} . E \varepsilon F:=L_{\mathrm{e}}\left(E_{\mathrm{co}}^{\prime}, F\right)$ is called Schwartz's $\varepsilon$-product of $E$ and $F$ $[29,39]$; here $e$ denotes the topology of uniform convergence on the equicontinuous subsets of $E^{\prime}$. The map $T \mapsto T^{t}$ is an isomorphism between $E \varepsilon F$ and $F \varepsilon E$. For more information, especially for the representation of spaces of vector-valued functions, we refer the reader to [5, 29, 38, 39]. In case $Y$ is a Fréchet-Schwartz (or (FS)) space, i.e. a Fréchet space which has a defining spectrum of Banach spaces with compact linking maps, $Y \varepsilon E=L_{\beta}\left(Y_{\beta}^{\prime}, E\right)$, since $Y$ is in particular a Montel space, i.e. a locally convex space which is barrelled and such that all its bounded sets are relatively compact. Montel spaces are reflexive and Fréchet-Schwartz spaces have even a fundamental system of reflexive Banach spaces (cf. [29, 33]). A (DFS)-space is the strong dual of a Fréchet-Schwartz space and can be represented as a countable inductive limit of a sequence of (reflexive) Banach spaces with compact linking maps.

A locally convex space $E$ is said to be locally complete whenever every absolutely convex, closed, bounded subset $B$ of $E$ spans a Banach space $E_{B}$ endowed with the Minkowski gauge of $B$. A linear subspace $F$ of $E$ is said to be locally closed if for every continuously embedded normed space $(X,\|\cdot\|)$ and every sequence $\left(x_{n}\right)_{n} \subseteq F \cap X$ which converges to some $x$ in the normed space $X$, we have $x \in F$. The local closure of a linear subspace $F \subseteq E$ is defined as the smallest locally closed subspace of $E$ which contains $F$, and it is denoted by $\bar{F}^{\text {lc }}$. For a locally convex space $E$, if $\widehat{E}$ denotes the completion of $E$, the local completion $E^{\text {lc }}$ of $E$ is defined as the local closure of $E$ in $\widehat{E}$. Every locally complete subspace of $E$ is locally closed, and a locally closed subspace of a locally complete space is locally complete [36, 5.1.20].

2.2. Holomorphic, harmonic and $\mathscr{C}^{\infty}$ functions. Our notation for spaces of (vector-valued) differentiable or holomorphic functions is standard. We refer the reader to $[4,27,37-39]$. 
A function $f: \Omega \subseteq \mathbb{R}^{N} \rightarrow E$ from an open connected subset (a domain) $\Omega$ of $\mathbb{R}^{N}$ into a locally convex space is said to be of class $\mathscr{C}^{1}$ if, for all $1 \leq i \leq N$, there is a continuous function $\partial f / \partial x_{i}: \Omega \rightarrow E$ such that

$$
\frac{\partial f(x)}{\partial x_{i}}=\lim _{t \rightarrow 0} \frac{1}{t}\left(f\left(x+t e_{i}\right)-f(x)\right), \quad x \in \Omega .
$$

Here $e_{i}$ denotes the $i$ th vector of the canonical basis of $\mathbb{R}^{N}$. For smooth functions on $\Omega \subseteq \mathbb{R}^{N}$ we use standard multi-index notation. Thus, if $\alpha=$ $\left(\alpha_{1}, \ldots, \alpha_{N}\right) \in \mathbb{N}^{N}$, then

$$
\partial^{\alpha} f=\frac{\partial^{|\alpha|}}{\partial x^{\alpha}} f=\frac{\partial^{|\alpha|}}{\partial x_{1}^{\alpha_{1}} \cdots \partial x_{N}^{\alpha_{N}}} f,
$$

where $|\alpha|=\alpha_{1}+\cdots+\alpha_{N}$. The space of all functions $f: \Omega \rightarrow E$ such that $\partial^{|\alpha|} f / \partial x^{\alpha}: \Omega \rightarrow E$ is a well defined continuous function for $|\alpha| \leq k$ is denoted by $\mathscr{C}^{k}(\Omega, E)$. Whenever $f: \Omega \rightarrow E$ is infinitely differentiable and $P(\partial, x)=\sum_{|\alpha| \leq m} a_{\alpha}(x) \partial^{\alpha}$ is a linear partial differential operator with smooth coefficients, then $P(\partial, x) f$ is also an infinitely differentiable function.

A function $f: \Omega \rightarrow E$ defined on an open subset $\Omega$ of the complex plane $\mathbb{C}$ is said to be holomorphic if, for each $z_{0} \in \Omega$, there exists $r>0$ and a sequence $\left(a_{n}\right)_{n} \subset E$ such that $f(z)=\sum_{n=0}^{\infty} a_{n}\left(z-z_{0}\right)^{n}$ for each $z \in B\left(z_{0}, r\right)$. The space of holomorphic functions with values in $E$ is denoted by $\mathscr{H}(\Omega, E)$. If the space $E$ is locally complete, a function $f \in \mathscr{C}^{\infty}(\Omega, E)$ belongs to $\mathscr{H}(\Omega, E)$ if and only if $f$ satisfies the Cauchy-Riemann equations. Analogously one can define the space of vector-valued harmonic functions $h(\Omega, E)$ as the vector-valued kernel of the Laplacian. Several variable vectorvalued holomorphic and harmonic functions are defined in a natural way.

If $\Omega$ is a domain and $E$ is locally complete, then the spaces $\mathscr{C}^{\infty}(\Omega, E)$ (resp. $\mathscr{H}(\Omega, E), \hbar(\Omega, E)$ ) and $\mathscr{C}^{\infty}(\Omega) \varepsilon E$ (resp. $\left.\mathscr{H}(\Omega) \varepsilon E, \hbar(\Omega) \varepsilon E\right)$ can be canonically identified via the map $f \mapsto T_{f}(u):=u \circ f, u \in E^{\prime}$. This is a consequence of [12, Prop. 2]. The result is well known when $E$ is quasicomplete (see [27, Theorem 16.7.4] and also [25, 38]).

2.3. Sheaves. We now recall the definition of topological sheaf. We refer the reader to [6, Section 1] or [35, Chapter V, Section 2] for more details and examples.

Definition 1. Let $\Omega$ be an open set in $\mathbb{R}^{N}$. We say that $\mathscr{F}$ is a sheaf of locally convex spaces over $\Omega$ if the following conditions are satisfied:

(a) For each open subset $U$ of $\Omega$ there is a locally convex space $\mathscr{F}(U)$ such that $\mathscr{F}(\emptyset):=\{0\}$ and there are continuous linear maps (called restrictions) $\varrho_{U, V}: \mathscr{F}(U) \rightarrow \mathscr{F}(V)$ for $V \subseteq U$ such that $\varrho_{U, W}=$ $\varrho_{V, W} \circ \varrho_{U, V}$ whenever $W \subseteq V \subseteq U$. 
(b) If $\omega \subseteq \Omega$ is open and $\omega$ is the union of open subsets $\{U: U \in \mathscr{U}\}$, then $\mathscr{F}(\omega)$ is the projective limit of $\left(\mathscr{F}(U), \varrho_{\omega, U}\right)_{U \in \mathscr{U}}$. In particular, for each family $\left\{f_{U} \in \mathscr{F}(U): U \in \mathscr{U}\right\}$ satisfying $\varrho_{U, U \cap V}\left(f_{U}\right)=$ $\varrho_{V, U \cap V}\left(f_{V}\right), U, V \in \mathscr{U}$, there is a unique $f \in \mathscr{F}(\omega)$ with $\varrho_{\omega, U}(f)=$ $f_{U}$ for each $U \in \mathscr{U}$.

REMARK 2. The open mapping theorem for (LB) spaces implies that if $\mathscr{F}$ is a sheaf over $\Omega$ of distinguished Fréchet spaces and $\left\{U_{n}: n \in \mathbb{N}\right\}$ is a countable open covering of an open subset $\omega$ of $\Omega$ then $\mathscr{F}(\omega)^{\prime}=$ $\operatorname{ind}_{n} \mathscr{F}\left(U_{n}\right)^{\prime}$, the inductive limit taken with respect to the transposes of the restrictions.

In the following we will omit "of locally convex spaces" when we refer to a sheaf. Our main example is the sheaf $\mathscr{C}^{\infty}(\Omega)$ of infinitely differentiable functions over a domain $\Omega$ : for every open set $U \subseteq \Omega$ the vector space $\mathscr{C}^{\infty}(U)$ is the vector space of all infinitely differentiable functions defined on $U$. Here the $\varrho_{U, V}$ are simply the restrictions. By a closed subsheaf of $\mathscr{C}^{\infty}$ over $\Omega$ we mean a sheaf $\mathscr{F}$ such that each $\mathscr{F}(\omega) \subseteq \mathscr{C}^{\infty}(\omega)$ is closed for each $\omega \subseteq \Omega$ open. The restriction maps are the same. All these sheaves are Fréchet-Schwartz sheaves; by this we mean that $\mathscr{F}(\omega)$ is a Fréchet-Schwartz space for each $\omega \subseteq \Omega$ open. We remark that some of our results could be formulated in a more abstract way so as to include other Fréchet-Schwartz sheaves of functions over $\Omega$, like the ultradifferentiable functions of Beurling type.

Vector-valued sheaves are defined using $\varepsilon$-products e.g. in $[6,1.4]$. Let $\Omega$ be a domain in $\mathbb{R}^{N}$ and $\mathscr{C}^{\infty}$ be the sheaf of infinitely differentiable functions. Let $P_{1}(\partial, x), \ldots, P_{m}(\partial, x)$ be linear partial differential operators with smooth coefficients on $\Omega$. Let $P_{\omega}: \mathscr{C}^{\infty} \rightarrow\left(\mathscr{C}^{\infty}\right)^{m}$ be defined by

$$
P_{\omega}: \mathscr{C}^{\infty}(\omega) \rightarrow \mathscr{C}^{\infty}(\omega)^{m}, \quad f \mapsto\left(P_{1}(\partial, x) f, \ldots, P_{m}(\partial, x) f\right),
$$

for $\omega \subseteq \Omega$ open. For a locally complete space $E$ and $\omega \subseteq \Omega$ we consider the maps

$$
P_{\omega} \varepsilon \text { id }: \mathscr{C}^{\infty}(\omega) \varepsilon E \rightarrow \mathscr{C}^{\infty}(\omega)^{m} \varepsilon E .
$$

These maps define a morphism in the category of sheaves of vector spaces over $\Omega$. Moreover, $\operatorname{ker}\left(P_{\omega}\right) \varepsilon E=\operatorname{ker}\left(P_{\omega} \varepsilon\right.$ id $)$ and the maps

$$
\begin{aligned}
\operatorname{ker}\left(P_{\omega}\right) \varepsilon E & \rightarrow\left\{f \in \mathscr{C}^{\infty}(\omega, E): P_{1}(\partial, x) f=\cdots=P_{m}(\partial, x) f=0\right\}, \\
T & \mapsto f_{T}, \quad f_{T}(x)=T\left(\delta_{x}\right), x \in \omega,
\end{aligned}
$$

define an isomorphism of sheaves. These remarks lead to the following definition.

Definition 3. Let $\Omega$ be a domain in $\mathbb{R}^{N}$, let $\mathscr{F}$ be a closed subsheaf of $\mathscr{C}^{\infty}$ over $\Omega$, and let $E$ be a locally complete space. Then the sheaf defined 
by

$$
\mathscr{F}(\omega, E):=\left\{x \mapsto T\left(\delta_{x}\right): T \in \mathscr{F}(\omega) \varepsilon E\right\}, \quad \omega \subseteq \Omega \text { open, }
$$

is called the sheaf of $\mathscr{F}$-functions with values in $E$.

This definition is coherent with the usual definition of the vector-valued sheaves of holomorphic and harmonic functions with values in a locally complete locally convex space $E$ by the remarks at the end of the former subsection. The coherence of this definition, which in principle could seem more restrictive, is also a direct consequence of Theorem 9 below. Observe that in the case of spaces of holomorphic or harmonic functions $\mathscr{F}$ on $\Omega$, the spaces are closed subsheaves of the sheaf $\mathscr{C}$ of continuous functions on $\Omega$. Equivalently, since all the spaces are Fréchet, the spaces $\mathscr{C}(\omega)$ and $\mathscr{C}^{\infty}(\omega)$ induce the same topology on $\mathscr{F}(\omega)$ for each $\omega \subseteq \Omega$ open. The same holds for sheaves defined by kernels of hypoelliptic linear partial differential operators with constant coefficients. The following definitions are needed to formulate precisely the first problem we want to deal with.

Definition 4. A set $M \subseteq \Omega \times \mathbb{N}_{0}^{N}$ is called a set of uniqueness for $\mathscr{F}(\Omega)$ if $g \in \mathscr{F}(\Omega)$ vanishes whenever $\partial^{\alpha} g(x)=0$ for all $(x, \alpha) \in M$, i.e. whenever $\operatorname{span}\left\{\delta_{x} \circ \partial^{\alpha}:(x, \alpha) \in M\right\}$ is $\sigma\left(\mathscr{F}(\Omega)^{\prime}, \mathscr{F}(\Omega)\right)$-dense.

Definition 5. If $M \subseteq \Omega \times \mathbb{N}_{0}^{N}$ is a set of uniqueness for $\mathscr{F}(\Omega)$ and $G \subseteq E^{\prime}$ is a separating subspace, we define $\mathscr{F}_{G}(M, E)$ as the space of all $f: M \rightarrow E$ such that for each $u \in G$ there is $f_{u} \in \mathscr{F}(\Omega)$ with $\partial^{\alpha} f_{u}(x)=$ $u \circ f(x, \alpha),(x, \alpha) \in M$. Since $M$ is supposed to be a set of uniqueness for $\mathscr{F}(\Omega)$, the functions $f_{u}$ are unique.

With the notation established so far, the first extension problem to be considered in this paper is as follows: When is the (injective) restriction map

$$
R_{M, G}: \mathscr{F}(\Omega, E) \rightarrow \mathscr{F}_{G}(M, E), \quad f \mapsto\left(\partial^{\alpha} f(x)\right)_{(x, \alpha) \in M},
$$

surjective?

3. Extension of vector-valued functions. Hereafter, $E$ denotes a locally complete locally convex space, $\Omega$ an open and connected subset of $\mathbb{R}^{N}, \mathscr{F}$ a closed subsheaf of $\mathscr{C}^{\infty}$ over $\Omega$ and $\mathscr{F}(\Omega, E)$ the corresponding sheaf of functions with values in $E$. Then $\mathscr{F}(\Omega)$ is an (FS)-space since it is supposed to be closed in $\mathscr{C}^{\infty}(\Omega)$. According to [2], a subspace $G \subseteq E^{\prime}$ is said to determine boundedness if every $\sigma(E, G)$-bounded subset of $E$ is also bounded in $E$. Clearly, if $G \subseteq E^{\prime}$ determines boundedness in $E^{\prime}$, then $G$ is separating, hence dense in $\left(E^{\prime}, \sigma\left(E^{\prime}, E\right)\right)$. The following lemma is very important in the rest of the article. It states known results in a way which is suitable for the applications we have in mind. 
LEMMA 6.

(a) If $T \in L(E, F)$, then there is a (unique) extension $T^{\mathrm{lc}} \in L\left(E^{\mathrm{lc}}, F^{\mathrm{lc}}\right)$ of $T$ to the local completions.

(b) If $Y$ is a Fréchet-Schwartz space and $X$ is a subspace of the (DFS) space $Y^{\prime}$, then the local closure of $X$ in $Y^{\prime}$ satisfies $\bar{X}^{\mathrm{lc}}=\bar{X}^{Y^{\prime}}$.

(c) If $E$ is a locally convex space and $t$ is an admissible topology, i.e. $\sigma\left(E, E^{\prime}\right) \leq t \leq \tau\left(E, E^{\prime}\right), \tau\left(E, E^{\prime}\right)$ being the Mackey topology, then $(E, t)^{\mathrm{lc}}=E^{\mathrm{lc}}$ algebraically. In particular, if $E$ is locally complete, then $E$ equipped with an admissible topology is also locally complete.

Proof. Part (a) is exactly [36, 5.1.25]. Part (b) is a consequence of Raikov's theorem $[36,8.5 .28]$. It is enough to show that the local closure $\bar{X}^{\mathrm{lc}}$ of $X$ is closed in $Y^{\prime}$. Since $Y$ is a Fréchet-Schwartz space, we have $Y^{\prime}=\operatorname{ind}_{n} G_{n}, G_{n}$ a Banach space and the linking maps $G_{n} \rightarrow G_{n+1}$ are compact for each $n \in \mathbb{N}$. The locally closed subspace $\bar{X}^{\text {lc }}$ intersects each $G_{n}$ in a closed subset of $G_{n}$. We can apply Raikov's theorem [36, 8.5.28] to conclude that $\bar{X}^{\text {lc }}$ is closed in $Y^{\prime}$. Part (c) follows from [36, 5.1.6 and 7], since

$$
\left(E, \tau\left(E, E^{\prime}\right)\right)^{\wedge} \hookrightarrow(E, t)^{\wedge} \hookrightarrow\left(E, \sigma\left(E, E^{\prime}\right)\right)^{\wedge}
$$

and all the admissible topologies have the same bounded sets.

In the rest of the article we will make the following natural identification: Suppose that $X$ is a dense locally convex subspace of the dual $Y^{\prime}$ of a Fréchet-Schwartz space $Y$. Since $Y$ is reflexive, we consider $Y$ as an algebraic subspace of $X^{\star}$. In fact, $Y$ is the set of all elements of the dual $X^{\star}$ of $X$ which are continuous on $X$ for the topology induced by $Y^{\prime}$. In case no locally convex topology is mentioned on $X$, we endow it with the finest locally convex topology which makes any linear mapping $T: X \rightarrow E$ continuous for an arbitrary locally convex space $E$. In this case $T^{t}$ acts from $E^{\prime}$ into $X^{\star}$.

Proposition 7. Let $Y$ be a Fréchet-Schwartz space, let $X \subseteq Y^{\prime}$ be a dense subspace, and let $E$ be a locally complete space. If $T: X \rightarrow E$ is a not necessarily continuous linear map, then the following conditions are equivalent:

(i) There is a (unique) extension $\widehat{T} \in L\left(Y^{\prime}, E\right)$ of $T$.

(ii) $T^{t}\left(E^{\prime}\right) \subseteq Y\left(=Y^{\prime \prime}\right)$.

(iii) $\left(T^{t}\right)^{-1}(Y)\left(=\left\{u \in E^{\prime}: u \circ T \in Y\right\}\right)$ determines boundedness in $E$.

Proof. Trivially (i) implies (ii) and (ii) implies (iii). Clearly, the map $T:(X, \sigma(X, Y)) \rightarrow\left(E, \sigma\left(E,\left(T^{t}\right)^{-1}(Y)\right)\right)$ is always continuous. If we assume (iii), the latter space is locally complete. Lemma 6 implies that the 
local completion of $(X, \sigma(X, Y))$ is $\left(Y^{\prime}, \sigma\left(Y^{\prime}, Y\right)\right)$ and so there is a unique continuous linear extension $\widehat{T}:\left(Y^{\prime}, \sigma\left(Y^{\prime}, Y\right)\right) \rightarrow\left(E, \sigma\left(E,\left(T^{t}\right)^{-1}(Y)\right)\right)$. Now, $Y^{\prime}$ endowed with its strong topology is a bornological space and $\widehat{T}: Y^{\prime} \rightarrow E$ maps bounded sets in $Y^{\prime}$ into bounded sets in $E$. Hence $\widehat{T}$ is continuous.

Corollary 8. Let $Y$ be a Fréchet-Schwartz space, $E$ a locally complete space, $T \in L\left(Y^{\prime}, E\right)$ and $F$ a locally closed subspace of $E$. If there exists a separating subspace $X$ of $Y^{\prime}$ such that $T(X) \subseteq F$ then $T \in L\left(Y^{\prime}, F\right)$.

Proof. The restriction $T: X \rightarrow F$ is continuous, hence there is a unique extension $T^{\mathrm{lc}}: X^{\mathrm{lc}} \rightarrow F^{\mathrm{lc}}=F$. Now $Y^{\prime}=X^{\mathrm{lc}}$ by Lemma $6(\mathrm{~b})$, so $T=T^{\mathrm{lc}}$.

THEOREM 9. Let $\mathscr{F}$ be a closed subsheaf of $\mathscr{C}^{\infty}$ over a domain $\Omega \subseteq \mathbb{R}^{N}$, let $M$ be a set of uniqueness for $\mathscr{F}(\Omega)$, and let $G$ be a subspace of the dual of a locally complete space $E$ which determines boundedness. Then the restriction map $R_{M, G}$ from $\mathscr{F}(\Omega, E)$ to $\mathscr{F}_{G}(M, E)$ is surjective.

Proof. Let $f \in \mathscr{F}_{G}(M, E)$. The space $X:=\operatorname{span}\left\{\delta_{x} \circ \partial^{\alpha}:(x, \alpha) \in M\right\}$ is a $\left(\right.$ weak $\left.^{\star}\right)$ dense subspace of the dual of the Fréchet-Schwartz space $Y:=\mathscr{F}(\Omega)$. Let the linear map $T: X \rightarrow E$ be determined by $T\left(\delta_{x} \circ \partial^{\alpha}\right):=$ $f(x, \alpha),(x, \alpha) \in M$. Since $G$ is $\sigma\left(E^{\prime}, E\right)$-dense, $T$ is well defined. Let $u \in G$ and $f_{u}$ be the unique element in $\mathscr{F}(\Omega)$ with $\partial^{\alpha} f_{u}(x)=u \circ T\left(\delta_{x} \circ \partial^{\alpha}\right)$, $(x, \alpha) \in M$. We can consider $f_{u}$ as a linear form on $X$, so we obtain $u \circ T \in \mathscr{F}(\Omega)=Y$ for all $u \in G$, hence $\left(T^{t}\right)^{-1}(Y)$ determines boundedness in $E$. By Proposition 7, there is an extension $\widehat{T} \in \mathscr{F}(\Omega) \varepsilon E$ of $T$. Putting $\widehat{f}(x):=\widehat{T}\left(\delta_{x}\right), x \in \Omega$, we conclude that $R_{M, G}(\widehat{f})=f$.

In particular, Theorem 9 shows that if $f: \Omega \rightarrow E$ is a function such that $u \circ f \in \mathscr{F}(\Omega)$ for each $u \in E^{\prime}$ then there exists $T: \mathscr{F}(\Omega)^{\prime} \rightarrow E$ such that $f(x)=T\left(\delta_{x}\right)$ for every $x \in \Omega$. Therefore, for $\Omega \subseteq \mathbb{C}^{N}=\mathbb{R}^{2 N}$ open, one can obtain directly from Theorem 9 the representation $\mathscr{H}(\Omega, E) \simeq \mathscr{H}(\Omega) \varepsilon E$ valid for locally complete spaces $E$ (cf. $[12,28]$ ). Moreover, to illustrate the scope of Theorem 9, we mention that it gives a direct proof of the fact that weak- $\mathscr{C}^{\infty}$ implies $\mathscr{C}^{\infty}$ : Let $f: \Omega \rightarrow E$ be a map into a locally complete space $E$ such that $u \circ f \in \mathscr{C}^{\infty}(\Omega)$ for all $u \in E^{\prime}$. Theorem 9 shows that $f(x)=T_{f}\left(\delta_{x}\right), x \in \Omega$, with $T_{f} \in \mathscr{C}^{\infty}(\Omega) \varepsilon E$. Using the Arzelà-Ascoli theorem the map $S: \Omega \rightarrow \mathscr{C}^{\infty}(\Omega)^{\prime}, x \mapsto \delta_{x}$, is infinitely differentiable, hence $f=T_{f} \circ S$ is infinitely differentiable. This also applies to holomorphic and harmonic functions. Thus, a general version of the Dunford-Grothendieck theorem is a simple consequence of our extension result, Theorem 9. Also to illustrate the applicability of Theorem 9 we mention explicitly how to extend two results obtained in [2] to holomorphic functions with values in Banach spaces $E$; it is enough to take as $\mathscr{F}$ the sheaf of holomorphic functions. 
Corollary 10.

(a) Let $\mathscr{F}$ be a closed subsheaf of $\mathscr{C}^{\infty}$ over a domain $\Omega \subseteq \mathbb{R}^{N}$ and let $E$ be a locally complete locally convex space. If $G \subseteq E^{\prime}$ determines boundedness in $E$ then $\mathscr{F}(\Omega, E)$ is formed by the functions $f: \Omega \rightarrow E$ such that $u \circ f \in \mathscr{F}(\Omega)$ for all $u \in G$.

(b) (cf. [2, Theorem 3.5, Lemma 3.6]) Let $E$ be a locally complete locally convex space. Let $\Omega \subseteq \mathbb{C}$ be a domain, $A \subseteq \Omega$ a set with an accumulation point, and $f: A \rightarrow E$ a map such that $u \circ f$ has an analytic extension to $\Omega$ for each u contained in a subspace $G$ of $E^{\prime}$ which determines boundedness on $E$. Then there exists $\widehat{f} \in \mathscr{H}(\Omega, E)$ which extends $f$.

(c) (cf. [2, Theorem 2.2]) Let $\Omega \subseteq \mathbb{C}$ be a domain, $E$ a locally complete space, $F$ a locally closed subspace of $E$, and $f \in \mathscr{H}(\Omega, E)$. Assume that either

(i) the set $\Omega_{0}:=\{z \in \Omega: f(z) \in F\}$ has an accumulation point in $\Omega$, or

(ii) there exists $z_{0} \in \Omega$ such that $\partial^{k} f\left(z_{0}\right) / \partial z^{k} \in F$ for $k=0,1,2, \ldots$. Then $f(z) \in F$ for all $z \in \Omega$.

Proof. Part (a) follows from Theorem 9 for $M=\Omega$. Part (b) is a direct consequence of Theorem 9. Part (c) follows from Corollary 8.

In fact, [28, Theorems 3 and 8] are immediate consequences of Theorem 9.

Remark 11. In [2, Theorem 3.5] it is shown that if $E$ is a Banach space and $G$ is a closed and almost norming subspace of $E^{\prime}$, which means that $E$ is a topological subspace of the Banach space $G^{\prime}$, then the conclusion of Corollary 10(b) holds. Such a subspace $G \subset E^{\prime}$ determines boundedness in $E$ by the uniform boundedness principle. Hence Corollary 10(b) is a proper extension of [2, Theorem 3.5]. We include an application of Corollary 10(b) which cannot be deduced from [2, Theorem 3.5].

Let $X, Y$ be Banach spaces. For $x \in X$ and $y \in Y^{\prime}$, we define $\delta_{x, y}$ : $L(X, Y) \rightarrow \mathbb{K}, T \mapsto y(T(x))$. The set $G:=\operatorname{span}\left\{\delta_{x, y}: x \in X, y \in Y^{\prime}\right\}$ determines boundedness in $L(X, Y)$ endowed with its norm topology. This is a consequence of the Banach-Steinhaus theorem. Therefore, as a consequence of Corollary 10(b), if $M$ is a subset of a domain $\Omega \subseteq \mathbb{C}$ with an accumulation point and $f: M \rightarrow L(X, Y)$ is a function such that $z \mapsto y(f(z)(x))$ has a holomorphic extension to $\Omega$ for each $y \in Y^{\prime}$ and $x \in X$, then $f$ has an extension $\widehat{f} \in \mathscr{H}(\Omega, L(X, Y))$, where $L(X, Y)$ is endowed with its norm topology. This extension result implies the well known fact that each $L(X, Y)$-valued holomorphic function for the weak operator topology is also holomorphic for the norm topology. 
It is worth remarking that [2, Theorem 1.5] shows that if $E$ is a Banach space, $\mathbb{D}$ is the unit disc in $\mathbb{C}$ and $G \subseteq E^{\prime}$ is a subspace which does not determine boundedness in $E$, then there exists a discontinuous function $f: \mathbb{D} \rightarrow E$ such that $u \circ f \in \mathscr{H}(\mathbb{D})$ for all $u \in G$. Hence Theorem 9 is optimal if we only require $M$ to be a set of uniqueness.

4. Extension of locally bounded functions. Let $\Omega$ be a domain in $\mathbb{C}$. A subset $M \subseteq \Omega$ is said to fix the topology in $\mathscr{H}(\Omega)$ (determine local convergence in [24]) if for all $K \subseteq \Omega$ compact there is $L \subseteq \Omega$ compact and $C \geq 1$ such that

$$
\sup _{z \in K}|g(z)| \leq C \sup _{z \in M \cap L}|g(z)| \quad \text { for all } g \in \mathscr{H}(\Omega) .
$$

Grosse-Erdmann [24] posed the following problem (see the end of Section 3 and comments below the statement of Theorem 2 in the Introduction of [24]): Let $M \subseteq \Omega$ fix the topology in $\mathscr{H}(\Omega)$, let $E$ be a locally convex space, let $f: M \rightarrow E$ be a map such that $f(M \cap K)$ is bounded in $E$ for all compact $K \subseteq \Omega$, and assume that for a separating subspace $G$ of $E^{\prime}, u \circ f$ has a holomorphic extension to $\Omega$ for every $u \in G$. Does $f$ have a holomorphic extension to $\Omega$ ? Gramsch [22] proved that this is so if $\bar{G}^{\beta\left(E^{\prime}, E\right)}=E^{\prime}$, which clearly includes the case that $E$ is semireflexive (cf. [28, Theorem 6]). His result inspired Grosse-Erdmann to study this problem and he gave a positive solution for $E$ being $B_{r}$-complete (see [24, Remark 2(b), p. 406]). Below we give a unified proof of these two cases, and show that the answer to the problem is in general negative.

The following definitions are needed to pose the problem in a more abstract form.

Definition 12. Let $Y$ be a Fréchet space. An increasing sequence $\left(B_{n}\right)_{n \in \mathbb{N}}$ of bounded subsets of $Y^{\prime}$ fixes the topology in $Y$ if $\left(B_{n}^{\circ}\right)_{n \in \mathbb{N}}$ is a fundamental system of zero neighbourhoods of $Y$.

Definition 13. Let $\Omega$ be a domain in $\mathbb{R}^{N}$, and let $\mathscr{F}$ be a closed subsheaf of $\mathscr{C}^{\infty}$ over $\Omega$. A subset $M$ of $\Omega \times \mathbb{N}_{0}^{N}$ fixes the topology in $\mathscr{F}(\Omega)$ if for every compact $K \subseteq \Omega$ and every $k \in \mathbb{N}$ there is a compact $L \subseteq \Omega, l \in \mathbb{N}$ and $C \geq 1$ such that

$$
\begin{aligned}
\sup \left\{\left|\partial^{\alpha} g(x)\right|: x \in K,\right. & |\alpha| \leq k\} \\
& \leq C \sup \left\{\left|\partial^{\alpha} g(x)\right|: x \in L,|\alpha| \leq l,(x, \alpha) \in M\right\}
\end{aligned}
$$

for all $g \in \mathscr{F}(\Omega)$.

For the space of holomorphic functions on $\Omega \subseteq \mathbb{C}$ the subsets $M \subseteq \Omega$ fixing the topology in $\mathscr{H}(\Omega)$ can be characterized by a nice geometrical property, as we see below (cf. [26, 2.5.2, 2.6.8]). 


\section{REMARK 14.}

(a) Let $\Omega \subseteq \mathbb{C}^{N}$ be a pseudo-convex domain. Then $M \subseteq \Omega$ fixes the topology in $\mathscr{H}(\Omega)$ if and only if the $\mathscr{H}(\Omega)$-hulls $\widehat{K \cap M}_{\Omega}, K \subseteq \Omega$ compact, are a fundamental system of compact subsets of $\Omega$.

(b) If $\Omega \subseteq \mathbb{C}$ is a domain, then it is pseudoconvex and the $\mathscr{H}(\Omega)$-hull of a compact subset $L \subseteq \Omega$ is the union of $L$ and the relatively compact components of $\Omega \backslash L$. Hence $M \subseteq \Omega$ fixes the topology in $\mathscr{H}(\Omega)$ (equivalently in $h(\Omega))$ if and only if there is a fundamental sequence $\left(O_{n}\right)_{n}$ of $\Omega$ of relatively compact open sets $O_{n}$ with $\partial O_{n} \subseteq \overline{O_{n+1} \cap M}$.

(c) In the case of $Y:=\mathscr{H}(\Omega)$ and $M \subseteq \Omega$ fixing the topology in $Y$, the constant $C$ in Definition 13 can be taken to be 1, because powers of holomorphic functions are holomorphic.

The cited question of Grosse-Erdmann becomes a special case of the following more general problem. Let $\left(L_{n}\right)_{n}$ be a fundamental sequence of compact (or relatively compact open) subsets of $\Omega$. Let $M \subseteq \Omega \times \mathbb{N}_{0}^{N}$. Set $M_{n}:=\left\{(x, \alpha) \in M: x \in L_{n},|\alpha| \leq n\right\}$ and $B_{n}:=\left\{\delta_{x} \circ \partial^{\alpha}:(x, \alpha) \in M_{n}\right\} \subseteq$ $\mathscr{F}(\Omega)^{\prime}$. Observe that a set $M$ fixes the topology in $\mathscr{F}(\Omega)$ in the sense of Definition 13 if and only if the sequence $\left(B_{n}\right)_{n}$ fixes the topology in $\mathscr{F}(\Omega)$ in the sense of Definition 12. This notation will be used in the rest of the article.

Let $M \subseteq \Omega \times \mathbb{N}_{0}^{N}$ fix the topology in $\mathscr{F}(\Omega)$ and let $G$ be a separating subspace of $E^{\prime}$. We define

$$
\mathscr{F}_{G}(M, E)_{\mathrm{lb}}:=\left\{f \in \mathscr{F}_{G}(M, E): f\left(M_{n}\right) \text { is bounded in } E \text { for } n \in \mathbb{N}\right\} .
$$

Observe that $R_{M, G}(f)$ belongs to $\mathscr{F}_{G}(M, E)_{\mathrm{lb}}$ for each $f \in \mathscr{F}(\Omega, E)$. In this terminology, the cited question of Grosse-Erdmann becomes a special case of the following more general problem: Let $\mathscr{F}(\Omega)$ be a closed subsheaf of $\mathscr{C}^{\infty}$ over $\Omega$, let $M \subseteq \Omega \times \mathbb{N}_{0}^{N}$ be a set which fixes the topology in $\mathscr{F}(\Omega)$, and let $G \subseteq E^{\prime}$ be a separating subspace. Is the (injective) restriction map

$$
R_{M, G}: \mathscr{F}(\Omega, E) \rightarrow \mathscr{F}_{G}(M, E)_{\mathrm{lb}}, \quad f \mapsto\left(\partial^{\alpha} f(x)\right)_{(x, \alpha) \in M},
$$

surjective?

Lemma 15. Let $Y$ be a Fréchet-Schwartz space, let $\left(U_{n}\right)_{n \in \mathbb{N}}$ be fundamental system of zero neighbourhoods for $Y$, and let $X \subseteq Y^{\prime}$ be a sequentially dense subspace. If $T: X \rightarrow E$ is a linear map into a locally convex space $E$ then

$$
\bigcap_{n \in \mathbb{N}} \operatorname{span}\left({\overline{\left(T^{t}\right)^{-1}\left(U_{n}\right)}}^{\sigma\left(E^{\prime}, E\right)}\right) \subseteq\left(T^{t}\right)^{-1}(Y) .
$$

Proof. It is clear that the space on the left side of $(*)$ does not depend on the choice of the fundamental system of zero neighbourhoods. Let 
$Y$ be the reduced projective limit of a sequence $\left(G_{n}\right)_{n \in \mathbb{N}}$ of reflexive Banach spaces such that there are compact linking maps $P_{n+1, n}: G_{n+1} \rightarrow G_{n}$ with dense range. We denote by $P_{n}$ the induced map from $Y$ to $G_{n}$. Then $Y^{\prime}=\operatorname{ind}_{n} G_{n}^{\prime}$ is the injective inductive limit of an increasing sequence of reflexive Banach spaces with compact inclusions $i_{n, n+1}: G_{n}^{\prime} \hookrightarrow G_{n+1}^{\prime}$, where $i_{n, n+1}:=\left(P_{n+1, n}\right)^{t}$. Using the Grothendieck factorization theorem $[33,24.33]$, the sequential density of $X$ and the fact that each convergent sequence in a (DFS)-space is convergent in one step, it is easy to see that we can assume that $X_{n}:=G_{n}^{\prime} \cap X$ is dense in $G_{n}^{\prime}$ for each $n \in \mathbb{N}$. Let $B_{n}$ be the unit ball of $G_{n}$. The sets $U_{n}:=P_{n}^{-1}\left(B_{n}\right), n \in \mathbb{N}$, form a fundamental system of zero neighbourhoods in $Y$. We can even assume that they form a zero basis. Take

$$
u \in \bigcap_{n \in \mathbb{N}} \operatorname{span}\left({\overline{\left(T^{t}\right)^{-1}\left(U_{n}\right)}}^{\sigma\left(E^{\prime}, E\right)}\right) .
$$

For each $n \in \mathbb{N}$ there is $\lambda_{n+1} \geq 1$ with $u \in \lambda_{n+1}{\overline{\left(T^{t}\right)^{-1}\left(U_{n+1}\right)}}^{\sigma\left(E^{\prime}, E\right)}$. So, there is a net $\left(u_{\alpha}^{n}\right)_{\alpha \in I} \subseteq E^{\prime}$ with $u_{\alpha}^{n} \circ T \in \lambda_{n+1} U_{n+1}, \alpha \in I$, such that $\left(u_{\alpha}^{n} \circ T(x)\right)_{\alpha \in I}$ converges to $u \circ T(x)$ for all $x \in X$. By the very definition of the $U_{n}$, for each $\alpha \in I$ there exists $v_{\alpha}^{n} \in Y$ such that $P_{n+1}\left(v_{\alpha}^{n}\right)(x)=u_{\alpha}^{n} \circ T(x)$ for each $x \in X_{n+1}$ and $P_{n+1}\left(v_{\alpha}^{n}\right) \in \lambda_{n+1} B_{n+1}$. Now the compactness of $P_{n+1, n}$ yields a subnet $\left(P_{n}\left(v_{\sigma(\beta)}^{n}\right)\right)_{\beta \in J}$ of $\left(P_{n}\left(v_{\alpha}^{n}\right)\right)_{\alpha \in I}=\left(P_{n+1, n}\left(P_{n+1}\left(v_{\alpha}^{n}\right)\right)\right)_{\alpha \in I}$ such that $\left(P_{n}\left(v_{\sigma(\beta)}^{n}\right)\right)_{\beta \in J}$ converges in the Banach space $G_{n}$ to $g_{n}$. But for each $x \in$ $X_{n} \subseteq X_{n+1}$ and for each $\alpha \in I$ we have $P_{n}\left(v_{\alpha}^{n}\right)(x)=P_{n+1}\left(v_{\alpha}^{n}\right)\left(i_{n, n+1}(x)\right)=$ $u_{\alpha}^{n} \circ T(x)$. Then $g_{n}(x)=u \circ T(x)$ for each $x \in X_{n}$ and for each $n \in \mathbb{N}$. The density of $X_{n}$ in $G_{n}^{\prime}$ yields $P_{n+1, n}\left(g_{n+1}\right)=g_{n}$ for each $n \in \mathbb{N}$. This means precisely $u \in\left(T^{t}\right)^{-1}(Y)$.

Theorem 16. Let $Y$ be a Fréchet-Schwartz space, let $\left(B_{n}\right)_{n \in \mathbb{N}}$ fix the topology in $Y$, and let $T: X:=\operatorname{span}\left(\bigcup\left\{B_{n}: n \in \mathbb{N}\right\}\right) \rightarrow E$ be a linear map into a locally complete space $E$ which is bounded on each $B_{n}$. If

(a) $\left(T^{t}\right)^{-1}(Y)$ is strongly dense in $E^{\prime}$ or

(b) $\left(T^{t}\right)^{-1}(Y)$ is weak $k^{\star}$-dense in $E^{\prime}$ and $E$ is $B_{r}$-complete,

then $T$ has a (unique) extension $\widehat{T} \in Y \varepsilon E$.

Proof. We first show that $X$ is sequentially dense in $Y^{\prime}$. The sequence $\left(B_{n}^{\circ \circ}\right)_{n \in \mathbb{N}}$ is a fundamental sequence of bounded sets in the (DFS)-space $Y^{\prime}$. Accordingly, for each $n$ there is $m>n$ such that the Banach space generated by $B_{m}^{\circ \circ}$ induces on $B_{n}^{\circ \circ}$ the topology $\sigma\left(Y^{\prime}, Y\right)$. By the bipolar theorem, the absolutely convex hull of $B_{n}$ is $\sigma\left(Y^{\prime}, Y\right)$-dense in $B_{n}^{\circ \circ}$, hence it is sequentially dense, since this topology is metrizable. As every element of $Y^{\prime}$ is in one of the sets $B_{n}^{\circ \circ}$, the proof is complete. 
(1) If $V_{n}:=T\left(B_{n}\right)^{\circ}, U_{n}:=B_{n}^{\circ}$, we obtain $V_{n} \cap\left(T^{t}\right)^{-1}(Y) \subseteq\left(T^{t}\right)^{-1}\left(U_{n}\right)$, $n \in \mathbb{N}$. We can apply Lemma 15 to get

$$
\bigcap_{n \in \mathbb{N}} \operatorname{span}\left({\overline{V_{n} \cap\left(T^{t}\right)^{-1}(Y)}}^{\sigma\left(E^{\prime}, E\right)}\right) \subseteq\left(T^{t}\right)^{-1}(Y) .
$$

(2) In view of Proposition 7 it is enough to show that $E^{\prime}=\left(T^{t}\right)^{-1}(Y)$. Now case (a) is trivial, since the $V_{n}$ are strong zero neighbourhoods and $\left(T^{t}\right)^{-1}(Y)$ is $\beta\left(E^{\prime}, E\right)$-dense. In the case of (b) we have to show that $\left(T^{t}\right)^{-1}(Y)$ is nearly closed, i.e. the weak ${ }^{\star}$-closure of the intersection of it with any equicontinuous set is contained in it. But this follows from the fact that each $V_{n}$ absorbs equicontinuous sets, since they are strong zero neighbourhoods. Thus, for each zero neighbourhood $U$ in $E, U^{\circ} \cap\left(T^{t}\right)^{-1}(Y) \subseteq$ $\operatorname{span}\left(V_{n} \cap\left(T^{t}\right)^{-1}(Y)\right)$ for each $n \in \mathbb{N}$. Taking weak ${ }^{\star}$-closures easily yields the conclusion.

Analyzing the previous proof we see that it is enough (instead of (a) or (b)) to ensure that $\left(T^{t}\right)^{-1}(Y)$ is weak ${ }^{\star}$-dense and has, in addition, the following property: for each decreasing sequence $\left(V_{n}\right)_{n \in \mathbb{N}}$ of strong zero neighbourhoods in $E^{\prime}$ with $(\star)$, one has $\left(T^{t}\right)^{-1}(Y)=E^{\prime}$.

TheOREM 17. If $M \subseteq \Omega \times \mathbb{N}_{0}^{N}$ fixes the topology in $\mathscr{F}(\Omega)$ and $G \subseteq E^{\prime}$ is separating, then the restriction map $R_{M, G}$ from $\mathscr{F}(\Omega, E)$ to $\mathscr{F}_{G}(M, E)_{\mathrm{lb}}$ is surjective in the following two cases:

(a) $E$ is a $B_{r}$-complete space or

(b) $E$ is locally complete and $G$ is strongly dense.

Proof. Let $f \in \mathscr{F}_{G}(M, E)_{\mathrm{lb}}$. There exists $f_{u} \in \mathscr{F}(\Omega)$ such that $u \circ$ $f(x, \alpha)=\partial^{\alpha} f_{u}(x)$ for each $(x, \alpha) \in M$ and for each $u \in G$. Then the linear map $T: \operatorname{span} \bigcup_{n} B_{n} \rightarrow E$ defined by $T\left(\delta_{x} \circ \partial^{\alpha}\right)=f(x, \alpha)$ is well defined and bounded on each $B_{n}$. The conclusion follows by applying Theorem 16 to $T$.

From Theorem 17 we obtain a general positive solution for the problem of Wrobel, valid for the harmonic case. That is, if $\Omega \subseteq \mathbb{C}^{N}$ is a domain and $E$ is a Banach space (or more generally a $B_{r}$-complete space), if $f: \Omega \rightarrow E$ is a locally bounded function such that $u \circ f$ is holomorphic or harmonic for each $u \in G \subseteq E^{\prime}$ separating then $f$ is holomorphic or harmonic. This can be applied to the following concrete result:

Let $H$ be a complex Hilbert space. For $x, y \in H$ we consider the continuous linear mappings $\delta_{x, y}: L(H) \rightarrow \mathbb{C}, T \mapsto\langle T x, y\rangle$. The subspace $G_{1}:=\operatorname{span}\left\{\delta_{x, y}: x, y \in H\right\} \subseteq L(H)^{\prime}$ determines boundedness in $L(H)$. This can be easily checked using the Banach-Steinhauss theorem (see Remark 11). The subspace $G_{2}=\operatorname{span}\left\{\delta_{x, x}: x \in H\right\} \subseteq L(H)^{\prime}$ coincides with $G_{1}$ 
by the polarization formula for sesquilinear forms. If $\left(e_{i}\right)_{i \in I}$ is an orthonormal basis in $H$ then $G_{3}=\operatorname{span}\left\{\delta_{e_{i}, e_{j}}: i, j \in I\right\} \subseteq L(H)^{\prime}$ is $\sigma\left(L(H)^{\prime}, L(H)\right)$ dense. Thus, in view of Corollary 10(a) and the above comment, we obtain the following result: Given $\Omega \subseteq \mathbb{C}$ open and $f: \Omega \rightarrow L(H)$, the following assertions are equivalent:

(1) $f \in \hbar(\Omega, L(H))$,

(2) $u \circ f \in h(\Omega)$ for each $u \in G_{1}$,

(3) $u \circ f \in \hbar(\Omega)$ for each $u \in G_{2}$,

(4) $f$ is locally bounded and $u \circ f \in \hbar(\Omega)$ for each $u \in G_{3}$.

This extends [17, Lemma 1] and hence contradicts [17, Example 1 and the previous assertion]. Grosse-Erdmann [24, Remark 1(d)] had already observed that [17, Example 1] was not correct. We also remark that if $H$ is a real Hilbert space then the space $G_{2}$ could not even be $\sigma\left(E^{\prime}, E\right)$-dense (cf. [33, Example 16.19]).

As an immediate consequence of Theorem 17, we obtain the following result, which is valid for harmonic and several variable holomorphic functions and extends [22, Satz 3.3] and [24, Theorem 2]. By saying that a closed subsheaf $\mathscr{F}$ of $\mathscr{C}^{\infty}$ over $\Omega$ satisfies the maximum principle we mean that $\max _{z \in K}|f(z)|=\max _{z \in \partial K}|f(z)|$ for each $f \in \mathscr{F}(\Omega)$ and for each $K \subseteq \Omega$ compact.

Corollary 18. Let $\mathscr{F}(\Omega)$ be closed in $\mathscr{C}(\Omega)$ and satisfy the maximum principle, and let $\left(O_{n}\right)_{n}$ be a fundamental sequence of relatively compact subdomains of $\Omega$ with $\partial O_{n} \subseteq \overline{M \cap O_{n+1}}$ for each $n$. If $f: M \rightarrow E$ is a function such that $f(M \cap K)$ is bounded in $E$ for each compact subset $K$ of $\Omega$ and $u \circ f$ admits an extension $f_{u} \in \mathscr{F}(\Omega)$ for each $u \in G \subseteq E^{\prime}$, then $f$ admits an extension $\widehat{f} \in \mathscr{F}(\Omega, E)$ whenever $E$ is $B_{r}$-complete and $G$ is separating (i.e. $\sigma\left(E^{\prime}, E\right)$-dense) or $E$ is locally complete and $G$ is $\beta\left(E^{\prime}, E\right)$ dense.

If $G \subseteq E^{\prime}$ determines boundedness in a locally complete space $E$ then $(E, \sigma(E, G))$ is a locally complete space. Moreover, if $S \subseteq E^{\prime}$ is dense in $G$ for the strong $\beta\left(E^{\prime}, E\right)$ topology then it is also dense for the $\beta(G, E)$ topology, since these two topologies coincide on $G$. This observation together with Corollary 10(a) yields the following result, which is relevant when $E=X^{\prime}$, $X$ a Banach space and $G$ a dense subspace of $X \subseteq E^{\prime}$.

REMARK 19. The conclusions of Theorem 17 and Corollary 18 remain true if $E$ is locally complete and $\bar{G}^{\beta\left(E^{\prime}, E\right)}$ determines boundedness in $E$.

The next example shows that the assertion of Corollary 18 is not true if $E$ is only assumed to be locally complete and $G$ is $\sigma\left(E^{\prime}, E\right)$-dense. 
EXAMPLE 20.

(a) Let $\Omega=\mathbb{C}$ and $M:=\bigcup_{n} \gamma_{n}$, where $\gamma_{n}:=\left\{n e^{i t}: t \in[0,2 \pi) \cap \mathbb{Q}\right\}$. Then there is a function $f: M \rightarrow \bigoplus_{n \in \mathbb{N}} l_{1}$ such that $f\left(\gamma_{n}\right)$ is bounded for each $n \in \mathbb{N}$ and there exists a weak ${ }^{\star}$-dense subspace $G \subseteq\left(\bigoplus_{n \in \mathbb{N}} l_{1}\right)^{\prime}$ such that $u \circ f$ admits a holomorphic extension to $\Omega$ for each $u \in G$ but $f$ is not continuous.

(b) Let $\Omega=\mathbb{C}$ and $M:=\bigcup_{n} \gamma_{n}$, where $\gamma_{n}:=\left\{n e^{i t}: t \in[0,2 \pi)\right\}$. Then there is a function $f: M \rightarrow \bigoplus_{n \in \mathbb{N}} l_{1}\left(\gamma_{n}\right)$ such that $f\left(\gamma_{n}\right)$ is bounded for each $n \in \mathbb{N}$ and there exists a weak $k^{\star}$-dense subspace $G \subseteq\left(\bigoplus_{n \in \mathbb{N}} l_{1}\left(\gamma_{n}\right)\right)^{\prime}$ such that $u \circ f$ admits a holomorphic extension to $\Omega$ for each $u \in G$ but $f$ is not continuous.

Proof. We show (a). The proof of (b) is analogous. Take an enumeration $\gamma_{n}=\left\{z_{i}^{n}: i \in \mathbb{N}\right\}$. First observe that the linear mapping $T: \bigoplus_{n} l_{1} \rightarrow$ $\mathscr{H}(\mathbb{C})^{\prime},\left(\alpha^{n}\right)_{n \in \mathbb{N}} \mapsto \sum_{n=1}^{\infty} \sum_{i=1}^{\infty} \alpha_{i}^{n} \delta_{z_{i}^{n}}$, is continuous and injective. The continuity is easily obtained from the fact that each $\gamma_{n}$ is relatively compact, and so $T$ maps bounded sets in the bornological space $\bigoplus_{n} l_{1}$ to bounded sets in $\mathscr{H}(\mathbb{C})^{\prime}$. To see that it is injective we suppose that there exists a nontrivial sequence $\left(\alpha^{n}\right)_{n}$ in $\bigoplus_{n} l_{1}$ such that $u:=\sum_{n=1}^{\infty} \sum_{i=1}^{\infty} \alpha_{i}^{n} \delta_{z_{i}^{n}}=0$ in $\mathscr{H}(\mathbb{C})^{\prime}$. Fix $n_{0}, i_{0} \in \mathbb{N}$ such that $\alpha_{i_{0}}^{n_{0}} \neq 0$ and $\alpha_{i}^{n}=0$ for each $n>n_{0}$ and for each $i \in \mathbb{N}$. We relabel the double sequence $\left(\alpha_{i}^{n} \delta_{z_{i}^{n}}\right)$ as $\left(\alpha_{k} \delta_{z_{k}}\right)_{k}$. We may assume that $\alpha_{1} \delta_{z_{1}}=\alpha_{i_{0}}^{n_{0}} \delta_{z_{i_{0}} n_{0}}$. Take $k_{0} \in \mathbb{N}$ such that $\sum_{k \geq k_{0}}\left|\alpha_{k}\right| \leq\left|\alpha_{1}\right| / 3$. The function $f(z):=\left(z+z_{1}\right) / 2 z_{1}$ satisfies $f\left(z_{1}\right)=1$ and $\left|f\left(z_{k}\right)\right|<1$ for each $k>1$. We get $j \in \mathbb{N}$ such that $\left|\sum_{k=2}^{k_{0}} \alpha_{k} f^{j}\left(z_{k}\right)\right|<\left|\alpha_{1}\right| / 3$. Therefore $\left|u\left(f^{j}\right)\right|>\left|\alpha_{1} / 3\right|$, a contradiction.

We set $E:=\bigoplus_{n} l_{1}$. We return to the first enumeration of each $\gamma_{n}$. Now we define $f: M=\bigcup_{n} \gamma_{n} \rightarrow E, z_{i}^{n} \mapsto\left(\alpha^{j}\right)_{j}$, each $\alpha^{j} \in l_{1}$ being the zero sequence except $\alpha^{n}$ which has the $i$ th coordinate 1 and zeros elsewhere. It is clear that $f$ is not continuous because the $l_{1}$-norm $\left\|f\left(z_{i}^{n}\right)-f\left(z_{j}^{n}\right)\right\|$ is 2 for $i \neq j$ and $n \in \mathbb{N}$. It is also clear that $f\left(\gamma_{n}\right)$ is bounded in $E$ for each $n \in \mathbb{N}$. Now the injectivity and continuity of $T$ imply that $G:=\{g \circ T: g \in \mathscr{H}(\mathbb{C})\}$ is a $\sigma\left(E^{\prime}, E\right)$-dense subspace of $E^{\prime}$. To conclude we observe that for each $g \in \mathscr{H}(\mathbb{C})$, the function $g \circ T \circ f: M \rightarrow \mathbb{C}$ extends to $g \in \mathscr{H}(\mathbb{C})$.

REMARK 21. (i) It is clear that the functions in the above example cannot be extended holomorphically to $\mathbb{C}$. Therefore, this example solves Grosse-Erdmann's extension problem in the negative. Further, the linear mapping $T$ in the above proof solves problems (a) and (b) in [24] also in the negative. In fact, $T$ cannot be surjective because it is an injective continuous linear mapping between two (LB)-spaces and $\mathscr{H}(\mathbb{C})^{\prime}$ is Montel but $\bigoplus_{n} l_{1}$ is not. 
(ii) Example 20 shows that [2, Corollary 3.7] was incorrectly stated, as was pointed out to Arendt and Nikolski by the present authors. It is easy to extract from the results of [2] a correct version of [2, Corollary 3.7], a universal counterexample to the stated version and a correct proof of [2, Corollary 3.8]. Arendt and Nikolski have carried this out in [3]. Let us see the contradiction between [2, Corollary 3.7] and Example 20. If we set $\Gamma_{n}:=\bigcup_{1 \leq k<n} \gamma_{k}, V_{n}:=B(0, n+1)$ (the open ball in $\mathbb{C}$ of radius $n+1$ centred at zero) for $n \in \mathbb{N}, E:=\bigoplus_{k \in \mathbb{N}} l_{1}$, and we consider the Banach space $E_{n}:=\bigoplus_{1<k<n} l_{1}$ endowed with its natural norm defined as the sum of the $l_{1}$-norms of components, then the restriction

$$
\left.f\right|_{\Gamma_{n}}: \Gamma_{n} \rightarrow E_{n+1}
$$

has image in the unit ball of $E_{n+1}$ for all $n \in \mathbb{N}$. The restriction of $T$ to each $E_{n}$ is continuous. Hence, for all $n \in \mathbb{N}$,

$$
G:=\left\{\left.g \circ T\right|_{E_{n+1}}: g \in \mathscr{H}(\mathbb{C})\right\}
$$

is a $\sigma\left(E_{n+1}^{\prime}, E_{n+1}\right)$-dense subspace of $E_{n+1}^{\prime}$ such that, for each $g \in \mathscr{H}(\mathbb{C})$, $\left.\left.g \circ T\right|_{E_{n+1}} \circ f\right|_{\Gamma_{n}}$ admits the holomorphic extension $\left.g\right|_{V_{n}}$ to $V_{n}$ and

$$
\sup _{z \in V_{n}}|g(z)| \leq \sup _{z \in \gamma_{n+1}}|g(z)| \leq \sup _{e \in B_{E_{n+1}}}|g \circ T|_{E_{n+1}}(e)\left|=\left\|\left.g \circ T\right|_{E_{n+1}}\right\|_{E_{n+1}^{\prime}} .\right.
$$

[2, Corollary 3.7] would imply that $\left.f\right|_{\Gamma_{n}}$ could be extended holomorphically to $f_{n} \in \mathscr{H}\left(V_{n}, E_{n+1}\right)$, but $\left.f\right|_{\Gamma_{n}}$ is not continuous.

(iii) Corollary 18 implies that [2, Corollary 3.8] is true, even for several variable holomorphic functions.

5. Wolff type results. The main tool in the proof of Grosse-Erdmann's theorem [24, Theorem 1] is Wolff's theorem [41], which we now state in a more functional-analytic way, as in the preliminaries of [24]: If $\Omega \subseteq \mathbb{C}$ is a domain, then for each $u \in \mathscr{H}(\Omega)^{\prime}$ there exists a sequence $\left(z_{i}\right)_{i}$ relatively compact in $\Omega$ and a sequence $\left(\alpha_{i}\right)_{i} \in l_{1}$ such that $u=\sum_{i=1}^{\infty} \alpha_{i} \delta_{z_{i}}$. Our goal in this section is to obtain similar representations for dual spaces $\mathscr{F}^{\prime}(\Omega)$ of closed subsheaves of $\mathscr{C}^{\infty}(\Omega)$ and to derive extension results from these representations. For further information about extensions of Wolff's original result we refer to [34, Sections 5.7.8 and 5.8], [40] and the references quoted there.

Let $Y$ be a Fréchet-Schwartz space and let $\left(B_{n}\right)_{n}$ be an increasing sequence of bounded subsets of $Y^{\prime}$. We introduce notation which will be useful in the rest of the article. For $n \in \mathbb{N}$, we denote by $l_{1}\left(B_{n}\right)$ the Banach space of all summable families with index set $B_{n}$. The linear map

$$
j_{n}: l_{1}\left(B_{n}\right) \rightarrow Y_{\beta}^{\prime}, \quad j_{n}\left((\alpha(b))_{b \in B_{n}}\right):=\sum_{b \in B_{n}} \alpha(b) b
$$


is well defined and continuous. We denote by $Y^{\prime}\left(B_{n}\right)$ the image $j_{n}$ endowed with the quotient norm. Clearly $Y^{\prime}\left(B_{n}\right)$ is a Banach space continuously embedded in $Y_{\beta}^{\prime}$. Finally, we set $Y^{\prime}\left(\left(B_{n}\right)_{n \in \mathbb{N}}\right):=\operatorname{ind}_{n} Y^{\prime}\left(B_{n}\right)$, which is an (LB)-space continuously included in $Y_{\beta}^{\prime}$.

REMARK 22. Let $Y$ be a Fréchet-Schwartz space. If $\left(B_{n}\right)_{n}$ is an increasing sequence of bounded subsets of $Y^{\prime}, E$ is a locally complete locally convex space and $T: \operatorname{span}\left(\bigcup_{n} B_{n}\right) \rightarrow E$ is a linear map such that $T$ is bounded on each $B_{n}$ and $\left(T^{t}\right)^{-1}(Y)$ is $\sigma\left(E^{\prime}, E\right)$-dense, then there exists a unique continuous linear extension $\widehat{T}: Y^{\prime}\left(\left(B_{n}\right)_{n}\right) \rightarrow E$ (compare with Theorem 16).

In view of this remark, our extension problem has a positive solution whenever $\left(B_{n}\right)_{n}$ is an increasing sequence of bounded subsets of $Y^{\prime}$ such that $Y^{\prime}\left(\left(B_{n}\right)_{n \in \mathbb{N}}\right)=Y_{\beta}^{\prime}$ topologically. We now characterize these sequences of bounded sets.

Proposition 23. Let $Y$ be a nuclear Fréchet space with an increasing fundamental system $\left(\|\cdot\|_{n}\right)_{n \in \mathbb{N}}$ of seminorms, and let $\left(B_{n}\right)_{n \in \mathbb{N}}$ be an increasing sequence of bounded subsets of $Y^{\prime}$. Then the following assertions are equivalent:

(i) $Y^{\prime}\left(\left(B_{n}\right)_{n \in \mathbb{N}}\right)=Y_{\beta}^{\prime}$.

(ii) For every $\mu \in Y^{\prime}$ there are $n \in \mathbb{N},\left(\mu_{\nu}\right)_{\nu \in \mathbb{N}} \in B_{n}^{\mathbb{N}}$, and $\left(\lambda_{\nu}\right)_{\nu \in \mathbb{N}} \in l_{1}$ such that

$$
\mu=\sum_{\nu=1}^{\infty} \lambda_{\nu} \mu_{\nu} .
$$

(iii) For every $k \in \mathbb{N}$ there are $n \in \mathbb{N},\left(\mu_{\nu}\right)_{\nu \in \mathbb{N}} \in B_{n}^{\mathbb{N}}$, and a decreasing zero sequence $\left(\varepsilon_{\nu}\right)_{\nu \in \mathbb{N}}$ such that

$$
\|f\|_{k} \leq \sup _{\nu \in \mathbb{N}} \varepsilon_{\nu}\left|\mu_{\nu}(f)\right| \quad \text { for every } f \in Y .
$$

Proof. (i) and (ii) are equivalent by the open mapping theorem.

For $k \in \mathbb{N}$, let $C_{k}$ denote the polar of the unit ball of the $k$ th seminorm. We denote by $E_{k}^{\prime}$ the Banach space spanned by $C_{k}$. Assume (i). By Grothendieck's factorization theorem the inductive spectra of $Y^{\prime}\left(\left(B_{n}\right)_{n \in \mathbb{N}}\right)$ and of $Y_{\beta}^{\prime}$ are equivalent. Since $Y$ is nuclear there is $n \in \mathbb{N}$ such that the inclusion $i_{k, n}: E_{k}^{\prime} \rightarrow Y^{\prime}\left(B_{n}\right)$ is nuclear. Now we observe the following two facts, which can be easily checked:

(a) Let $E$ be a Banach space and let $I$ be an index set. If $S: E \rightarrow l_{1}(I)$ is a nuclear linear map and $B$ is the unit ball of $E$ then there exists $\beta=(\beta(i))_{i \in I} \in l_{1}(I)$ such that

$$
S(B) \subseteq\left\{(\lambda(i))_{i \in I}:|\lambda(i)| \leq|\beta(i)|, i \in I\right\} .
$$


(b) If $E$ is a Banach space, $F$ is a Hausdorff quotient of a Banach space $G$ and $T: E \rightarrow F$ is a nuclear linear map then there exists a nuclear linear map $S: E \rightarrow G$ such that $T=p \circ S$, where $p$ is the quotient map.

We apply (a) and (b) to $i_{k, n}$ to obtain $\left(\mu_{\nu}\right)_{\nu \in \mathbb{N}} \in B_{n}^{\mathbb{N}}$ and $\left(\beta_{\nu}\right)_{\nu \in \mathbb{N}} \in l_{1}$ such that

$$
C_{k} \subseteq\left\{\sum_{\nu=1}^{\infty} \lambda_{\nu} \mu_{\nu}:\left|\lambda_{\nu}\right| \leq \beta_{\nu}, \nu \in \mathbb{N}\right\} .
$$

If we choose a decreasing zero sequence $\left(\varepsilon_{\nu}\right)_{\nu \in \mathbb{N}}$ such that $C:=\sum_{\nu=1}^{\infty}\left|\beta_{\nu} / \varepsilon_{\nu}\right|$ $<\infty$, we obtain

$$
\|f\|_{k} \leq \sup \left\{\sum_{\nu=1}^{\infty} \lambda_{\nu} \mu_{\nu}(f):\left|\lambda_{\nu}\right| \leq \beta_{\nu}, \nu \in \mathbb{N}\right\} \leq C \sup _{\nu \in \mathbb{N}} \varepsilon_{\nu}\left|\mu_{\nu}(f)\right|
$$

for every $f \in Y$.

Assume now (iii) and fix $k \in \mathbb{N}$. Then $C_{k}$ is contained in the closure $D$ of the absolutely convex hull of $\left\{\varepsilon_{\nu} \mu_{\nu}: \nu \in \mathbb{N}\right\}$, and from [36, 3.2.13] we get $D=\left\{\sum_{\nu=1}^{\infty} \lambda_{\nu} \varepsilon_{\nu} \mu_{\nu}: \sum_{\nu=1}^{\infty}\left|\lambda_{\nu}\right| \leq 1\right\}$. This shows (ii).

REMARK 24. (a) Nuclearity plays an important role in the proof of the equivalence between (ii) and (iii), but (i) and (ii) are equivalent for distinguished Fréchet spaces. In our setting, $\mathscr{F}(\Omega)$ is a nuclear space because it is a subspace of $\mathscr{C}^{\infty}(\Omega)$.

(b) Notice that if $Y^{\prime}\left(\left(B_{n}\right)_{n \in \mathbb{N}}\right)=Y_{\beta}^{\prime}$ then $\left(B_{n}\right)_{n}$ fixes the topology in the Fréchet-Schwartz space $Y$, because the polar of the unit ball of $Y^{\prime}\left(B_{n}\right)$ in $Y$ coincides with the polar of $B_{n}$ for each $n \in \mathbb{N}$. Example 20 together with Remark 21(i) shows that $\left(B_{n}\right)_{n}$ fixing the topology in $Y$ is not enough to have the equality $Y^{\prime}\left(\left(B_{n}\right)_{n \in \mathbb{N}}\right)=Y^{\prime}$.

To obtain concrete examples of Wolff descriptions we introduce the following notation. Let $\mathscr{F}$ be a closed subsheaf of $\mathscr{C}^{\infty}$ over an open set $\Omega \subseteq \mathbb{R}^{N}$ and let $U \subseteq V \subseteq \Omega$ be open sets. If $B \subseteq \mathscr{F}(U)^{\prime}$ we write $\left.B\right|_{\mathscr{F}(V)}:=\left\{u \circ \varrho_{V, U}: u \in B\right\}=\left(\varrho_{V, U}\right)^{t}(B) \subseteq \mathscr{F}(V)^{\prime}$.

LEMma 25. Let $\mathscr{F}$ be a closed subsheaf of $\mathscr{C}^{\infty}$ over an open domain $\Omega \subseteq \mathbb{R}^{N}$, let $\left(U_{n}\right)_{n}$ be an increasing covering of $\Omega$ by open subsets, and let $\left(B_{j}^{n}\right)_{j} \subseteq \mathscr{F}\left(U_{n}\right)^{\prime}$ be a sequence of bounded sets which fixes the topology in $\mathscr{F}\left(U_{n}\right)$ for $n \in \mathbb{N}$. Assume also that $\left.\left(B_{j}^{n}\right)\right|_{\mathscr{F}\left(U_{n+1}\right)} \subseteq B_{j}^{n+1}$ and that $B_{n}:=\left.\bigcup_{j}\left(B_{j}^{n}\right)\right|_{\mathscr{F}(\Omega)}$ is bounded in $\mathscr{F}(\Omega)^{\prime}$ for each $n \in \mathbb{N}$. Suppose that $T: \operatorname{span}\left(\bigcup_{n} B_{n}\right) \rightarrow E$ is a linear mapping into a locally complete space $E$ such that $\left(T^{t}\right)^{-1}(\mathscr{F}(\Omega))$ is separating and that, for all $n \in \mathbb{N}$, there exists a Banach space $E_{n} \hookrightarrow E$ continuously embedded such that $T\left(B_{n}\right)$ is bounded in $E_{n}$. Then there exists an extension $\widehat{T} \in \mathscr{F}(\Omega) \varepsilon E$ of $T$. 
Proof. First we observe that $\operatorname{span}\left(\bigcup_{n} B_{n}\right)$ is dense in $\mathscr{F}(\Omega)^{\prime}$ and so $\left(T^{t}\right)^{-1}(\mathscr{F}(\Omega))$ is meaningful. This can be easily deduced from the description of $\mathscr{F}(\Omega)$ as a projective limit of the spaces $\mathscr{F}\left(U_{n}\right)$ with respect to the restrictions, using the density of $\operatorname{span}\left(\bigcup_{j} B_{j}^{n}\right)$ in $\mathscr{F}\left(U_{n}\right)^{\prime}$. For $n \in \mathbb{N}$, we define $T_{n}: \operatorname{span}\left(\bigcup_{j} B_{j}^{n}\right) \rightarrow E_{n}, \mu \mapsto T\left(\left.\mu\right|_{F(\Omega)}\right)$. By the construction, $T_{n}\left(B_{j}^{n}\right)$ is bounded in $E_{n}$ for each $j \in \mathbb{N}$ and the subspace $H_{n}:=\left(T_{n}^{t}\right)^{-1}\left(\mathscr{F}\left(U_{n}\right)\right)$ of $E_{n}^{\prime}$ contains $\left.\left(T^{t}\right)^{-1}(\mathscr{F}(\Omega))\right|_{E_{n}}$. Hence $H_{n}$ is $\sigma\left(E_{n}^{\prime}, E_{n}\right)$-dense. Theorem 16(b) implies that there exists a continuous linear extension $\widehat{T}_{n}: \mathscr{F}\left(U_{n}\right)^{\prime} \rightarrow$ $E_{n} \hookrightarrow E$ of $T_{n}$. We observe that $\left.\widehat{T}_{n+1} \circ\left(\varrho_{U_{n+1}, U_{n}}\right)^{t}\right|_{\operatorname{span}\left(\cup_{j} B_{j}^{n}\right)}=T_{n}$ and that $\operatorname{span}\left(\bigcup_{j} B_{j}^{n}\right)$ is dense in $\mathscr{F}\left(U_{n}\right)^{\prime}$ for each $n \in \mathbb{N}$. Therefore, we apply Remark 2 to define $\widehat{T}: \mathscr{F}(\Omega)^{\prime}=\operatorname{ind}_{n} \mathscr{F}\left(U_{n}\right)^{\prime} \rightarrow E$ by $\widehat{T}(b)=\widehat{T}_{n}(b)$ whenever $b \in \mathscr{F}\left(U_{n}\right)^{\prime}$. Then $\widehat{T}$ is the desired extension of $T$.

The following abstract Wolff type result is now a consequence of the previous extension lemma.

THEOREM 26. Let $\mathscr{F}$ be a closed subsheaf of $\mathscr{C}^{\infty}$ over an open set $\Omega \subseteq$ $\mathbb{R}^{N}$, let $\left(U_{n}\right)_{n}$ be an increasing covering of $\Omega$ by open subsets, and let $\left(B_{j}^{n}\right)_{j} \subseteq$ $\mathscr{F}\left(U_{n}\right)^{\prime}$ be a sequence of bounded sets which fixes the topology in $\mathscr{F}\left(U_{n}\right)$ for $n \in \mathbb{N}$. Assume also that $\left.\left(B_{j}^{n}\right)\right|_{\mathscr{F}\left(U_{n+1}\right)} \subseteq B_{j}^{n+1}$ and that $B_{n}:=\left.\bigcup_{j}\left(B_{j}^{n}\right)\right|_{\mathscr{F}(\Omega)}$ is bounded in $\mathscr{F}(\Omega)^{\prime}$. Then $\mathscr{F}(\Omega)^{\prime}=\mathscr{F}(\Omega)^{\prime}\left(\left(B_{n}\right)_{n \in \mathbb{N}}\right)$ (topologically).

Proof. Set $E:=Y^{\prime}\left(\left(B_{n}\right)_{n \in \mathbb{N}}\right)$ and $E_{n}:=Y^{\prime}\left(B_{n}\right)$. We consider the inclusion $T: \operatorname{span}\left(\bigcup_{n} B_{n}\right) \rightarrow E$. Since $E \hookrightarrow \mathscr{F}(\Omega)^{\prime}$ continuously it follows that $\mathscr{F}(\Omega)$ is $\sigma\left(E^{\prime}, E\right)$-dense. We apply Lemma 25 to obtain a continuous linear map $\widehat{T}: \mathscr{F}(\Omega)^{\prime} \rightarrow E$ which extends $T$. But $\operatorname{span}\left(\bigcup_{n} B_{n}\right)$ is dense in $\mathscr{F}(\Omega)^{\prime}$. Hence the continuous inclusion $E \hookrightarrow \mathscr{F}(\Omega)^{\prime}$ is surjective. The topological equality follows from Proposition 23.

We see below that we can obtain Wolff type results for not necessarily increasing coverings by open sets.

Corollary 27. Let $\mathscr{F}$ be a closed subsheaf of $\mathscr{C}^{\infty}$ over an open domain $\Omega \subseteq \mathbb{R}^{N}$, let $\left(U_{n}\right)_{n}$ be a covering of $\Omega$ by open subsets, and let $\left(B_{j}^{n}\right)_{j} \subseteq$ $\mathscr{F}\left(U_{n}\right)^{\prime}$ be a sequence of bounded sets which fixes the topology in $\mathscr{F}\left(U_{n}\right)$ for $n \in \mathbb{N}$. Assume that $B_{n}:=\left.\bigcup_{j}\left(B_{j}^{n}\right)\right|_{\mathscr{F}(\Omega)}$ is bounded in $\mathscr{F}(\Omega)^{\prime}$ for each $n \in \mathbb{N}$. Then for each $\mu \in \mathscr{F}(\Omega)^{\prime}$ there exist $\left(\alpha_{i}\right)_{i} \in l_{1}, k \in \mathbb{N}$ and $\left(\mu_{i}\right)_{i} \subseteq$ $\bigcup_{1 \leq j \leq k} B_{j}$ such that $\mu=\sum_{i} \alpha_{i} \mu_{i}$.

Proof. We show that, if $U, V$ are two open subsets of $\Omega,\left(B_{j}^{U}\right)_{j}$ is a sequence of bounded subsets of $\mathscr{F}(U)^{\prime}$ which fixes the topology in $\mathscr{F}(U)$, $\left(B_{j}^{V}\right)_{j}$ is a sequence of bounded subsets of $\mathscr{F}(V)^{\prime}$ which fixes the topology in $\mathscr{F}(V)$ and we define $C_{n}:=\left.\left.B_{n}^{U}\right|_{\mathscr{F}(U \cup V)} \cup B_{n}^{V}\right|_{\mathscr{F}(U \cup V)}$, then $\left(C_{n}\right)_{n}$ is a sequence of bounded subsets of $\mathscr{F}(U \cup V)^{\prime}$ which fixes the topology in 
$\mathscr{F}(U \cup V)$. Since $\mathscr{F}$ is a sheaf, $\mathscr{F}(U \cup V)$ is the projective limit of the spaces $\mathscr{F}(U)$ and $\mathscr{F}(V)$ with respect to the restrictions $\varrho_{U \cup V, U}$ and $\varrho_{U \cup V, V}$. This implies that a fundamental system of zero neighbourhoods is given by the sets

$$
W_{n}:=\left(\varrho_{U \cup V, U}\right)^{-1}\left(\left(B_{n}^{U}\right)^{\circ}\right) \cap\left(\varrho_{U \cup V, V}\right)^{-1}\left(\left(B_{n}^{V}\right)^{\circ}\right), \quad n \in \mathbb{N} .
$$

It is straightforward to show that $W_{n}=C_{n}^{\circ}$. Now the conclusion can be obtained by applying Theorem 26 to the covering $\left(V_{n}\right)_{n}$ of $\Omega$ defined by $V_{n}:=\bigcup_{1 \leq j \leq n} U_{j}$

From Theorem 26 we also have the following corollary.

COROLlary 28. Let $\mathscr{F}$ be a closed subsheaf of $\mathscr{C}^{\infty}$ over an open domain $\Omega \subseteq \mathbb{R}^{N}$. If $M \subseteq \Omega \times \mathbb{N}_{0}^{N}$ has the property that there exists an increasing countable covering $\left(U_{k}\right)_{k}$ of $\Omega$ by relatively compact open sets such that the sets $M_{n}:=\left\{(x, \alpha) \in M: x \in U_{n},|\alpha| \leq n\right\}$ fix the topology in $\mathscr{F}\left(U_{n}\right)$, $n \in \mathbb{N}$, then $\mathscr{F}(\Omega)^{\prime}=\mathscr{F}(\Omega)^{\prime}\left(\left(M_{n}\right)_{n \in \mathbb{N}}\right)$. Then the restriction map $R_{M, G}$ : $\mathscr{F}(\Omega, E) \rightarrow \mathscr{F}_{G}(M, E)_{\mathrm{lb}}$ is surjective for each locally complete locally convex space $E$ and for each weak $k^{\star}$-dense subspace $G$ of $E^{\prime}$.

We now list some examples satisfying the hypothesis of Corollary 28.

EXAMPLE 29.

(a) Any sheaf $\mathscr{F}$ of smooth functions which is closed in the sheaf of continuous functions $\mathscr{C}$ over $\Omega \subseteq \mathbb{R}^{N}$ and $M:=\Omega$. For any covering $\left(U_{k}\right)_{k}$ of $\Omega$ by relatively compact open subsets, $M_{k}=U_{k}$ fixes the topology in $\mathscr{F}\left(U_{k}\right)$.

(b) The sheaf $\mathscr{H}$ of holomorphic functions over $\Omega \subseteq \mathbb{C}^{N}$, and $M:=\Omega \backslash K$, $K \subseteq \Omega$ compact. For any increasing covering $\left(U_{k}\right)_{k}$ of $\Omega$ by relatively compact open subsets such that $K \subseteq U_{1}$ the sets $M_{k}:=M \cap U_{k}$ fix the topology of $\mathscr{H}\left(U_{k}\right)$ (cf. [24, Corollary 1]).

(c) The sheaf $\mathscr{H}$ of holomorphic functions over $\Omega:=\mathbb{C}^{N}, N \in \mathbb{N}$, and $M:=\bigcup_{k, n \in \mathbb{N}} S(0, k-1 / n)$. For $U_{k}:=B(0, k), k \in \mathbb{N}$, the sets $M_{k}:=M \cap U_{k}$ fix the topology $\mathscr{H}\left(U_{k}\right)$ (here $B(a, r)$ and $S(a, r)$ denote the ball and the sphere centered and $a$ with radius $r$ respectively). Example 20 shows that $M:=\bigcup_{k} S(0, k)$ does not satisfy the hypothesis of Corollary 28 in the one variable case.

We also have the following consequences:

(i) In the three examples above, Corollary 28 can be formulated in the following way. For each $\mu \in \mathscr{F}(\Omega)^{\prime}$ there exist $k \in \mathbb{N}$, a sequence $\left(z_{\nu}\right)_{\nu}$ in $M_{k}$ and a sequence $\left(\alpha_{\nu}\right)_{\nu} \in l_{1}$ such that

$$
\mu=\sum_{\nu=1}^{\infty} \alpha_{\nu} \delta_{z_{\nu}} .
$$


Hence, if $E$ is a locally complete space, $G$ is a weak ${ }^{\star}$-dense subspace of $E^{\prime}$ and $f: M \rightarrow E$ is a function such that $f(M \cap K)$ is bounded in $E$ for each compact subset $K$ of $\Omega$ and $u \circ f$ admits an extension in $\mathscr{F}(\Omega)$ for all $u \in G$ then there exists an extension $\widehat{f} \in \mathscr{F}(\Omega, E)$ of $f$. We also remark that in (b) and (c) one can take the sheaf of harmonic functions on $\Omega \subseteq \mathbb{C}$ instead of the sheaf of holomorphic functions. Thus, we have proper extensions of [24, Theorem 1, Corollary 1].

(ii) Example 29(a) and Proposition 23 together imply that for each compact subset $K$ of the open set $\Omega \subset \mathbb{C}^{N}$, there exists a decreasing zero sequence $\left(\varepsilon_{\nu}\right)_{\nu}$ of positive numbers and a relatively compact sequence $\left(z_{\nu}\right)_{\nu} \subseteq \Omega$ such that, for each $f \in \mathscr{H}(\Omega)$,

$$
\sup _{z \in K}|f(z)| \leq \sup _{\nu \in \mathbb{N}} \varepsilon_{\nu}\left|f\left(z_{\nu}\right)\right| .
$$

In case $\Omega=\mathbb{C}^{N}$, Example 29 shows that for each $K$ compact there exists $k_{0} \in \mathbb{N}$ such that the sequence $\left(z_{\nu}\right)_{\nu}$ can be even taken in the set $\bigcup_{1 \leq k \leq k_{0}} \bigcup_{n \in \mathbb{N}} S(0, k-1 / n)$. Again we remark that there is no $k_{0} \in \mathbb{N}$ such that the sequence above could be taken in $\bigcup_{k \leq k_{0}} S(0, k)$. This is a consequence of Example 20 and Proposition 23.

Finally, we obtain Wolff type results for closed subsheaves $\mathscr{F}(\Omega, E)$ of $\mathscr{C}^{\infty}(\Omega, E)$ with $E$ Fréchet. To do this, we consider in these spaces the natural topology of uniform convergence of the derivatives on compact subsets of $\Omega$, which makes it a Fréchet space. This topology coincides with the one induced by the $\varepsilon$-product $\mathscr{F}(\Omega) \varepsilon E$. We refer to $[27,16.7]$ for the proof of this fact for the sheaf of one variable holomorphic functions.

Proposition 30. Let $\mathscr{F}$ be a closed subsheaf of $\mathscr{C}^{\infty}$ over an open set $\Omega \subseteq \mathbb{R}^{N}$. Let $\left(B_{n}\right)_{n}$ be an increasing sequence of bounded subsets of $\mathscr{F}(\Omega)^{\prime}$ such that $\mathscr{F}(\Omega)^{\prime}=\mathscr{F}(\Omega)^{\prime}\left(\left(B_{n}\right)_{n \in \mathbb{N}}\right)$. Let $E$ be a Fréchet space. For each $\mu \in \mathscr{F}(\Omega, E)^{\prime}$ there exists a sequence $\left(\alpha_{k}\right)_{k} \in l_{1}, n_{0} \in \mathbb{N}$, a sequence $\left(b_{k}\right)_{k} \subseteq$ $B_{n_{0}}$ and a bounded sequence $\left(v_{k}\right)_{k} \subseteq E^{\prime}$ such that

$$
\mu(f)=\sum_{k=1}^{\infty} \alpha_{k} b_{k}\left(v_{k} \circ f\right) \quad \text { for each } f \in \mathscr{F}(\Omega, E) .
$$

Proof. The space $\mathscr{F}(\Omega)$ is nuclear and hence $\mathscr{F}(\Omega)$ has the approximation property. Moreover, $\mathscr{F}(\Omega)$ is also separable. Thus, there exists a projective spectrum $\left(H_{n}\right)_{n}$ of separable Hilbert spaces such that $\mathscr{F}(\Omega)$ is its reduced projective limit. Let $E$ be the reduced projective limit of a sequence $\left(E_{n}\right)_{n}$ of Banach spaces. We have

$$
\mathscr{F}(\Omega, E)=\mathscr{F}(\Omega) \widehat{\otimes}_{\varepsilon} E=\operatorname{proj}_{n} H_{n} \widehat{\otimes}_{\varepsilon} E_{n} .
$$

We can apply $[14,16.6]$ to obtain

$$
\mathscr{F}(\Omega, E)^{\prime}=\operatorname{ind}_{n} H_{n}^{\prime} \widehat{\otimes}_{\pi} E_{n}^{\prime} .
$$


algebraically. Let $\mu \in \mathscr{F}(\Omega, E)^{\prime}$. There exists $k_{0} \in \mathbb{N}$ such that $\mu \in$ $H_{k_{0}}^{\prime} \widehat{\otimes}_{\pi} E_{k_{0}}^{\prime}$. Therefore, there exists a bounded sequence $\left(h_{k}\right)_{k}$ in $H_{k_{0}}^{\prime} \hookrightarrow$ $\mathscr{F}(\Omega)^{\prime}$, a bounded sequence $\left(e_{k}\right)_{k}$ in $E_{k_{0}}^{\prime} \hookrightarrow E$ and a sequence $\left(\lambda_{k}\right)_{k}$ in $l_{1}$ such that

$$
\mu(f)=\sum_{k=1}^{\infty} \lambda_{k}\left(h_{k} \otimes v_{k}\right)(f)=\sum_{k=1}^{\infty} \lambda_{k} h_{k}\left(v_{k} \circ f\right) .
$$

Since $\left(h_{k}\right)_{k}$ is bounded in $\mathscr{F}(\Omega)^{\prime}=\mathscr{F}(\Omega)^{\prime}\left(\left(B_{n}\right)_{n \in \mathbb{N}}\right)$, there exists $n_{0} \in \mathbb{N}$ such that $\left(h_{k}\right)_{k}$ is bounded in $\mathscr{F}(\Omega)^{\prime}\left(B_{n_{0}}\right)$. Hence we can get a sequence $\left(b_{i}\right)_{i} \subseteq B_{n_{0}}$ and $M>0$ such that for each $k \in \mathbb{N}$ there exists $\left(\alpha_{i}^{k}\right)_{i} \in l_{1}$ such that $\sum_{i}\left|\alpha_{i}^{k}\right|<M$ and $h_{k}=\sum_{i} \alpha_{i}^{k} b_{i}$. Therefore, for each $f \in \mathscr{F}(\Omega, E)$,

$$
\mu(f)=\sum_{k=1}^{\infty} \lambda_{k}\left(h_{k} \otimes v_{k}\right)(f)=\sum_{k=1}^{\infty} \lambda_{k} h_{k}\left(v_{k} \circ f\right)=\sum_{k=1}^{\infty} \sum_{i=1}^{\infty} \lambda_{k} \alpha_{i}^{k} b_{i}\left(v_{k} \circ f\right) .
$$

Relabelling the double series gives the desired formula.

By Example 29(a), in the sheaf $\mathscr{H}$ of holomorphic functions over $\Omega \subseteq \mathbb{C}^{N}$, for each $\mu \in \mathscr{H}(\Omega, E)^{\prime}$, there exist $\left(\alpha_{k}\right)_{k} \in l_{1},\left(v_{k}\right)_{k} \subseteq E^{\prime}$ bounded and $\left(z_{k}\right)_{k} \subseteq \Omega$ relatively compact such that, for each $f \in \mathscr{H}(\Omega, E)$,

$$
\mu(f)=\sum_{k=1}^{\infty} \alpha_{k} v_{k}\left(f\left(z_{k}\right)\right) .
$$

\section{References}

[1] E. Albrecht and J. Eschmeier, Analytic functional models and local spectral theory, Proc. London Math. Soc. (3) 75 (1997), 323-348.

[2] W. Arendt and N. Nikolski, Vector-valued holomorphic functions revisited, Math. Z. 234 (2000), 777-805.

[3] —, 一, Addendum: Vector-valued holomorphic functions revisited, Math. Z. 252 (2006), 687-689.

[4] W. Arendt, C. J. K. Batty, M. Hieber and F. Neubrander, Vector-Valued Laplace Transforms and Cauchy Problems, Birkhäuser, Basel, 2001.

[5] K. D. Bierstedt, Tensor products of weighted spaces, Bonner Math. Schriften 81 (1975), 26-58.

[6] K. D. Bierstedt, B. Gramsch und R. Meise, Approximationseigenschaft, Lifting und Kohomologie bei lokalkonvexen Produktgarben, Manuscripta Math. 19 (1976), 319364.

[7] K. D. Bierstedt and S. Holtmanns, Weak holomorphy and other weak properties, Bull. Soc. Roy. Sci. Liège 72 (2004), 377-381.

[8] W. M. Bogdanowicz, Analytic continuation of holomorphic functions with values in a locally convex space, Proc. Amer. Math. Soc. 22 (1969), 660-666.

[9] J. Bonet, Representaciones de los espacios $O_{M}(E)$ y $D_{L^{p}}(E)$, Collect. Math. 33 (1982), 23-41. 
[10] J. Bonet, P. Domański and M. Lindström, Weakly compact composition operators on weighted vector-valued Banach spaces of analytic mappings, Ann. Acad. Sci. Fenn. Math. 26 (2001), 233-248.

[11] J. Bonet and M. Friz, Weakly compact composition operators on locally convex spaces, Math. Nachr. 245 (2002), 26-44.

[12] J. Bonet, E. Jordá and M. Maestre, Vector-valued meromorphic functions, Arch. Math. (Basel) 79 (2002), 353-359.

[13] J. F. Colombeau, Differential Calculus and Holomorphy, North-Holland, Amsterdam, 1982.

[14] A. Defant and K. Floret, Tensor Norms and Operator Ideals, North-Holland, Amsterdam, 1993.

[15] S. Dineen, Complex Analysis on Infinite Dimensional Spaces, Springer, New York, 1999.

[16] N. Dunford, Uniformity in linear spaces, Trans. Amer. Math. Soc. 44 (1938), 305356.

[17] P. Enflo and L. Smithies, Harnack's theorem for harmonic compact operator-valued functions, Linear Algebra Appl. 336 (2001), 21-27.

[18] K. J. Engel and R. Nagel, One-Parameter Semigroups for Linear Evolution Equations, Grad. Texts in Math. 194, Springer, 2000.

[19] J. Eschmeier and M. Putinar, Spectral Decompositions and Analytic Sheaves, Clarendon Press, Oxford, 1996.

[20] - - - Spherical contractions and interpolation problems on the unit ball, J. Reine Angew. Math. 542 (2002), 219-236.

[21] B. Gramsch, Über das Cauchy-Weil-Integral für Gebiete mit beliebigem Rand, Arch. Math. (Basel) 28 (1977), 409-421.

[22] —, Ein Schwach-Stark-Prinzip der Dualitätstheorie lokalkonvexer Räume als Fortsetzungsmethode, Math. Z. 156 (1977), 217-230.

[23] K.-G. Grosse-Erdmann, The Borel Okada theorem revisited, Habilitation, Fernuniv. Hagen, 1993.

[24] -, A weak criterion for vector-valued holomorphy, Math. Proc. Cambridge Philos. Soc. 136 (2004), 399-411.

[25] A. Grothendieck, Sur certains espaces de fonctions holomorphes, J. Reine Angew. Math. 192 (1953), 35-64.

[26] L. Hörmander, An Introduction to Complex Analysis in Several Variables, NorthHolland, 1990.

[27] H. Jarchow, Locally Convex Spaces, Teubner, Stuttgart, 1981.

[28] E. Jordá, Extension of vector-valued holomorphic and meromorphic functions, Bull. Belg. Math. Soc. 12 (2005), 5-21.

[29] G. Köthe, Topological Vector Spaces I, II, Springer, Berlin, 1969, 1979.

[30] A. Kriegl and P. W. Michor, The Convenient Setting of Global Analysis, Amer. Math. Soc., Providence, RI, 1997.

[31] J. Laitila and H. O. Tylli, Composition operators on vector-valued harmonic functions and Cauchy transforms, preprint 411, Dept. Math., Univ. Helsinki, 2005.

[32] P. Liu, E. Saksman and H. O. Tylli, Small composition operators on analytic vectorvalued function spaces, Pacific J. Math. 184 (1998), 295-309.

[33] R. Meise and D. Vogt, Introduction to Functional Analysis, Clarendon Press, Oxford, 1997.

[34] N. K. Nikolski, Operators, Functions, and Systems: an Easy Reading, Vol. 1, Amer. Math. Soc., Providence, 2002. 
[35] V. P. Palamodov, Linear Differential Operators with Constant Coefficients, Springer, Berlin, 1970.

[36] P. Pérez Carreras and J. Bonet, Barrelled Locally Convex Spaces, North-Holland, Amsterdam, 1987.

[37] W. Rudin, Real and Complex Analysis, 3rd ed., McGraw-Hill, New York, 1991.

[38] L. Schwartz, Espaces des fonctions différentiables à valeurs vectorielles, J. Anal. Math. 4 (1954/55), 88-148.

[39] —, Théorie des distributions à valeurs vectorielles I-II, Ann. Inst. Fourier (Grenoble) 7 (1957), 1-141; 8 (1959), 1-209.

[40] R. V. Sibilev, A uniqueness theorem for Wolff-Denjoy series, Algebra i Analiz 7 (1995), 170-199 (in Russian); English transl.: St. Petersburg Math. J. 7 (1996), $145-168$.

[41] J. Wolff, Sur les séries $\sum \frac{A_{k}}{z-\alpha_{k}}$, C. R. Acad. Sci. Paris 173 (1921), 1327-1328.

[42] V. Wrobel, Analytic functions into Banach spaces and a new characterization for isomorphic embeddings, Proc. Amer. Math. Soc. 85 (1982), 539-543.

Departamento de Matemática Aplicada

and IMPA-UPV

ETS Arquitectura

Universidad Politécnica de Valencia

E-46071 Valencia, Spain

E-mail: jbonet@mat.upv.es

Departamento de Matemática Aplicada

and IMPA-UPV

E. Politécnica Superior de Alcoy

Universidad Politécnica de Valencia

Plaza Ferrándiz y Carbonell 2

E-03801 Alcoy (Alicante), Spain

E-mail: ejorda@mat.upv.es
FB IV - Mathematik

Universität Trier

D-54286 Trier, Germany

E-mail: frerick@uni-trier.de

Received May 11, 2006

Revised version September 4, 2007 